\title{
AN AUTOMATIC TRANSMITTER FOR DISTRESS SIGNALS*
}

\author{
By
}

\author{
Chester M. Agner \\ (Sacramento, California)
}

The instrument here described is principally for the purpose of giving ships that are not required by law to carry a radio apparatus a means of notifying other ships by radio telegraphy of their need for assistance, and of their position, in case of disability or disaster. This is accomplished without any assistance from a skilled operator, or one having any knowledge of radio telegraphy, or of the code used. There are at this moment many ships plying the oceans unprotected insofar as radio telegraphy may be considered a protection. They are unable to call for assistance and to advise other ships of their position in case of disaster or disability.

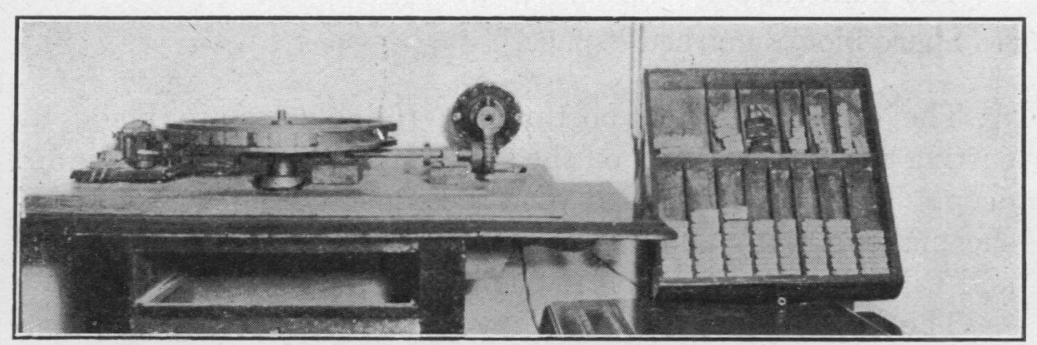

The apparatus mentioned consists of a brass disc, and a set of brass type blocks to be used to form a message. The disc is arranged to revolve at a speed of about 2.25 revolutions per minute, being driven by a small electric motor. The disc has a channel cut around its circumference with marginal internal flanges extending into the channel for the purpose of holding the type blocks.

The blocks are curved so as to conform to the curvature

* Received by the Editor, February 16, 1917. 
of the channel of the disc. They have two longitudinal grooves on opposite sides to receive the flanges, so that the blocks cannot escape. The grooves on the blocks are of such depth that a portion of the blocks projects radially outward beyond the outer edge of the disc. On one side of each type block is stamped the information, or its abbreviation, which the block is able to transmit.

The blocks shown in the figure consist of :-

Two-S O S blocks.

One--Ship's call letters.

Abbreviation of word "longitude" (lng).

Abbreviation of word "latitude" (lt.)

Abbreviation of word "east" (E).

Abbreviation of word "west" (W).

Abbreviation of word "north" (N).

Abbreviation of word "south" (S).

Signal (D) meaning "fire."

Signal (K) meaning "disabled."

Signal (C) meaning "in life boats."

Signal (L) meaning "on rocks."

The numerals.

Space blocks marked "space."

The non-conducting portions of the blocks are made by cutting grooves crosswise on the surface about 0.125 inch $(3 \mathrm{~mm}$.) deep. These cuts are filled with a composition of wax (such as shoemaker's wax) and rosin. This composition is fairly hard and will adhere perfectly if in the proper proportion.

The surface of the blocks having the conducting and nonconducting portions, will be brushed by a needle when the blocks are carried by the disc as it rotates, causing the opening and closing of a battery circuit operating a relay. This acts as a key in the primary circuit of a transformer.

Electrical connection between the disc and the relay is made by the needle brush and also by making connection at the base of the perpendicular axle upon which the disc turns. Grease or oil for lubrication is not used on this axle.

To form a message, it is necessary to select the desired blocks, placing them in the channel of the disc. The flanges of the disc at a certain point are broken away, so that the blocks can be 
inserted into the channel This break in the flanges is long enough to receive the longest block. The blocks can be placed on the flanges only on the right side of the entering place, because of the obstruction of a permanent stop member at the left end of the entrance. As one flange extends further out into the channel than the other, and as one groove of the block is made deeper than the other, it is impossible to insert blocks into the channel backwards.

This apparatus is designed to transmit automatically the position of a ship in case of distress, therefore the message must be arranged according to a certain form, and the spacing between words and letters, etc., is so arranged on the blocks as to comply with this form.

Form of message:-

(SOS) (SOS) (Ship's name) (longitude) $148^{\circ} 17^{\prime}(\mathrm{W})$ (latitude) $24^{\circ} 51^{\prime}(\mathrm{N})$ (Fire).

Words between parentheses are single blocks.

Rule:-Place a space block (marked "space") between degrees and minutes in both longitude and latitude: $\operatorname{lng} 148 \quad 17 \mathrm{~W}$. lt $24 \quad 51 \mathrm{~N}$.

The spacing between words, letters, or numbers, etc., is arranged on the blocks in such manner that they space automatically with the exception given in the above rule. It can therefore be seen that it is a very simple matter to set up a message. Altho this apparatus has blocks only for sending a position in latitude and longitude, other blocks may be added, such as the alphabet, so that a brief message could be formed giving the position of a ship as "so many miles from a certain point." This, of course, is not necessary.

When forming a message, the first block as it is placed in the channel should be pushed along until it is stopped by a projection in its path, at the point where the blocks were inserted into the channel. Each following block should be pushed along likewise until it is stopped by the block preceding it. When all the blocks forming the message have been placed on the flanges, the space between the last block and the part where the flanges are omitted should be filled with blank blocks having a non-conducting outer surface. These blocks are used to make the disc almost evenly balanced, so that when the ship rolls or if there is a heavy list, the motor will have a continuous even pull. If these blank blocks are not used, and the apparatus is not on a level, the motor will have a tendency to slow up and then speed as the disc revolves. 
A block having a set screw is placed on the flanges at the end of the row of blocks, its purpose being to prevent the blocks from having longitudinal movement. The opposite end from the set screw block is held by means of the permanent stop member projecting into the channel.

SUMMARY : A rotating disc is arranged to carry curved blocks on its periphery. Each block permits transmitting a certain sign. Simple distress messages can thus be sent by unskilled persons. 